\title{
Relationship between survivin expression and recurrence, and prognosis in hepatocellular carcinoma
}

\author{
Chao-Ping Ye, Cheng-Zhi Qiu, Zhong-Xin Huang, Qi-Chen Su, Wei Zhuang, Rui-Lan Wu, Xin-Feng Li
}

Chao-Ping Ye, Cheng-Zhi Qiu, Wei Zhuang, Xin-Feng Li, Department of Hepatobiliary Surgery, Second Affiliated Hospital of Fujian Medical University, Quanzhou 362000, Fujian Province, China

Zhong-Xin Huang, Qi-Chen Su, Rui-Lan Wu, Department of Pathology, Second Affiliated Hospital of Fujian Medical University, Quanzhou 362000, Fujian Province, China

Correspondence to: Chao-Ping Ye, Department of Hepatobiliary Surgery, Second Affiliated Hospital of Fujian Medical University, Quanzhou 362000, Fujian Province,

China.xulina9995@yahoo.com.cn

Telephone: +86-595-22780153 Fax: +86-595-22793591

Received: June 15, $2007 \quad$ Revised: September 2, 2007

\begin{abstract}
AIM: To study the expression of the inhibitor of apoptosis protein survivin in hepatocellular carcinoma (HCC), and its correlation with clinicopathological factors, cell proliferation, recurrence and prognosis after hepatectomy.
\end{abstract}

METHODS: Immunohistochemical staining of survivin and Ki-67 was performed by the standard streptavidinperoxidase technique on paraffin sections of 55 cases of HCC.

RESULTS: The positive rate of survivin in HCC was $52.7 \%(29 / 55)$. Significant correlation was found between survivin expression with portal vein thrombi and intrahepatic matastasistic nodes $(P<0.05)$. The recurrent rate in survivin-positive HCC was significantly higher than that in survivin-negative HCC after hepatectomy, the 1- and 3-year survival rate in patients with survivin-positive tumors was significantly lower than that in patients with survivin-negative tumors (58.62 and $10.34 \%$ vs 76.92 and $30.77 \%, P<0.05$, log-rank test). The proliferation index (Ki-67) in survivin-positive HCC $(33.83 \% \pm 18.90 \%)$ was significantly higher than that in survivin-negative HCC $(19.60 \% \pm 19.35 \%)(P<0.05)$.

CONCLUSION: Survivin may play an important role in progression of HCC by promoting cell proliferation, and may be positively correlated with high risk of disease recurrence and poor prognosis in HCC. Its expression may serve as a prognostic factor for patients with HCC after hepatectomy.

(c) 2007 WJG. All rights reserved.
Key words: Hepatocelluar carcinoma; Survivin; Proliferation index; Prognosis; Immunohistochemistry

Ye CP, Qiu CZ, Huang ZX, Su QC, Zhuang W, Wu RL, Li XF. Relationship between survivin expression and recurrence, and prognosis in hepatocellular carcinoma. World $J$ Gastroenterol 2007; 13(46): 6264-6268

http://www.wjgnet.com/1007-9327/13/6264.asp

\section{INTRODUCTION}

Although surgical resection is the most important method for hepatocellular carcinoma (HCC), the recurrent rates may be as high as $50 \%$ at 2 years after hepatectomy ${ }^{[1]}$. The recurrence of HCC may be related to a variety of factors, including biological markers. Molecular prognostic markers are likely to be of greatest benefit in the effective management of patients with HCC, however, these factors have not yet been sufficiently defined in patients with a high risk of cancer recurrence.

Survivin is a recently described member of the family of inhibitor of apoptosis proteins (IAPs). Recently, it has been shown that survivin is strongly associated with apoptosis, cell proliferation and cellcycle control $^{[2-5]}$. Survivin plays a crucial role in the genesis and progression of malignancy and is an important prognostic parameter in tumors ${ }^{[0-10]}$. This study investigated the expression of survivin in HCC and its correlation with clinicopathological factors, cell proliferation and prognosis.

\section{MATERIALS AND METHODS}

\section{Materials}

Tissue samples were obtained between December 2000 and December 2003 from 55 patients with HCC (41 men, 14 women; $24-74$ years old, mean age, 48.65 years). There were 27 patients with stage I - II , and 28 with stage IIIIV cancer. None of the patients received radiotherapy, chemotherapy or immunotherapy before surgery.

\section{Reagents}

Rabbit anti-human survivin polyclonal antibody was purchased from Santa Cruz Biotechnology (Santa Cruz, CA, USA). Mouse anti-human Ki-67 monoclonal antibody 


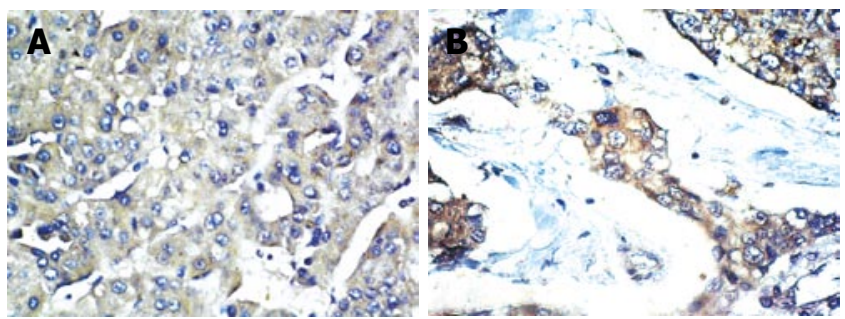

Figure 1 A: Positive expression of survivin in HCC (SP, $\times 200)$; B: Positive expression of survivin in HCC $(\mathrm{SP}, \times 400)$.

(MBI.1), streptavidin-peroxidase (SP) staining kit and diaminobenzidine (DAB) kit were supplied by Maixin-Bio, Fuzhou, China.

\section{Methods}

Expression of survivin and Ki-67 was detected using SP immunohistochemistry. Briefly, after deparaffinization and rehydration, antigen retrieval was accomplished by incubation in $0.01 \mathrm{~mol} / \mathrm{L}$ citric acid buffer ( $\mathrm{pH}$ 6.0), boiling for $1 \mathrm{~min}$ in a pressure cooker, and cooling and washing in tap water. The sections were incubated with hydrogen peroxide for $10 \mathrm{~min}$ and washed in PBS. Nonspecific reactions were blocked by incubation in a solution containing normal serum. The sections were incubated with a primary antibody (anti-survivin or Ki-67 antibody) overnight at $4^{\circ} \mathrm{C}$. The working dilution of anti-survivn antibody was 1:100. The sections were rinsed with PBS, and then incubated for $10 \mathrm{~min}$ at room temperature with biotinylated secondary antibody. After washing, streptavidin-biotin complex conjugated to horseradish peroxidase was applied for $10 \mathrm{~min}$ at room temperature. After three rinses with PBS, the sections were incubated with $\mathrm{DAB}$, rinsed with distilled water, counterstained with hematoxylin, and dehydrated and coverslipped. The sections were prepared for microscopy. Colorectal cancer tissues were used as a positive control. As a negative control, PBS was used to replace primary antibody.

\section{Scoring criteria for survivin expression}

Intensity and percentage of positive cells were used to evaluate each tissue section. The mean percentage of positive tumor cells and normal epithelial cells in at least five areas at $\times 400$ magnification was determined and assigned to one of five categories: $0,<5 \% ; 1,5 \%-24 \% ; 2$, $25 \%-49 \% ; 3,50 \%-74 \%$; and $4, \geqslant 75 \%$. The intensity of survivin immunostaining was scored as 0 (achromatic), 1 (light yellow), 2 (yellow), and 3 (brown). The percentage of positive cells and staining intensity were multiplied to produce a weighted score for each case. Cases with weighted scores $<1$ were defined as negative; all others were defined as positive.

\section{Determination of the Ki-67 proliferation index}

At least five high-power fields were chosen randomly in each section, and 500 cells were counted for each field. The $\mathrm{Ki}-67$ proliferation index was defined as the number of $\mathrm{Ki}$ 67-positive nuclei divided by the total number of colorectal cancer cells counted, and was expressed as a percentage.
Table 1 Correlation between survivin expression and clinicopathology in $\mathrm{HCC} n(\%)$

\begin{tabular}{|c|c|c|c|c|c|}
\hline \multirow{2}{*}{ Clinicopathological factor } & & \multirow{2}{*}{$n$} & \multicolumn{2}{|c|}{ Survivin expression } & \multirow{2}{*}{$P$ value } \\
\hline & & & positive & negative & \\
\hline \multirow[t]{2}{*}{ Sex } & Male & 41 & 23 & 6 & \multirow{2}{*}{0.392} \\
\hline & Female & 14 & 6 & 8 & \\
\hline \multirow[t]{2}{*}{ Age (yr) } & $\leqslant 55$ & 40 & 22 & 18 & \multirow{2}{*}{0.581} \\
\hline & $>55$ & 15 & 7 & 8 & \\
\hline \multirow[t]{3}{*}{ Tumor site } & Right lobe & 30 & 14 & 16 & \multirow{3}{*}{0.615} \\
\hline & Left lobe & 20 & 12 & 8 & \\
\hline & Whole liver & 5 & 3 & 2 & \\
\hline \multirow[t]{2}{*}{ HBsAg } & Positive & 42 & 21 & 21 & \multirow{2}{*}{0.467} \\
\hline & Negative & 13 & 8 & 5 & \\
\hline \multirow[t]{2}{*}{ Differentiation } & $\begin{array}{l}\text { Moderate to } \\
\text { well }\end{array}$ & 40 & 23 & 17 & \multirow[t]{2}{*}{0.247} \\
\hline & Poor & 15 & 6 & 9 & \\
\hline Intrahepatic & $(+)$ & 21 & 16 & 5 & \multirow{2}{*}{0.006} \\
\hline metastatic nodes & $(-)$ & 34 & 13 & 21 & \\
\hline \multirow[t]{2}{*}{ Portal vein thrombi } & $(+)$ & 14 & 12 & 2 & \multirow{2}{*}{0.004} \\
\hline & $(-)$ & 41 & 17 & 24 & \\
\hline \multirow[t]{2}{*}{ Tumor capsule } & $(+)$ & 22 & 12 & 10 & \multirow{2}{*}{0.825} \\
\hline & $(-)$ & 33 & 17 & 16 & \\
\hline \multirow[t]{2}{*}{ Tumor size $(\mathrm{cm})$} & $\leqslant 5$ & 16 & 8 & 8 & \multirow{2}{*}{0.795} \\
\hline & $>5$ & 39 & 21 & 18 & \\
\hline \multirow[t]{2}{*}{$\operatorname{AF} P(\mu \mathrm{g} / L)$} & $<400$ & 19 & 7 & 12 & \multirow{2}{*}{0.086} \\
\hline & $\geqslant 400$ & 36 & 22 & 14 & \\
\hline \multirow[t]{2}{*}{ Hepatocirrhosis } & $(+)$ & 37 & 21 & 16 & \multirow{2}{*}{0.391} \\
\hline & $(-)$ & 18 & 8 & 10 & \\
\hline \multirow[t]{2}{*}{ Tumor stage } & I - II & 27 & 14 & 13 & \multirow{2}{*}{0.898} \\
\hline & III-IV & 28 & 15 & 13 & \\
\hline
\end{tabular}

\section{Statistical analysis}

The survival curves were assessed by the Kaplan-Meier method and compared by a log-rank test. The $\chi^{2}$ test was performed for enumeration data comparison, and the $t$ test was used for comparison of measurement data. $P<0.05$ was considered statistically significant. All data analysis was performed with commercially available statistical analysis software packages (SSPS 11.5, SSPS, Chicago, IL, USA).

\section{RESULTS}

\section{Relationship between expression of survivin and clinical pathology}

Survivin protein expressed as brown-yellow particles in the cytoplasm after staining, and only one expressed both in the cytoplasm and nucleus after staining. The positive staining rate for survivin in the cytoplasm and nuclei was 29/55 (52.7\%) (Figure 1). There was a significant correlation between survivin expression and portal vein thrombi and intrahepatic metastatic nodes $(P<0.05)$. However, it was not related to the following factors: age and sex of the patient, tumor location, tumor differentiation, tumor size, presence of tumor capsule, clinical stage, complicating liver cirrhosis, preoperative alpha fetoprotein (AFP) level, and hepatitis B surface antigen (HBsAg) (Table 1). These findings suggest that the expression of survivin may be significantly associated with metastasis.

\section{Relationship between expression of survivin and proliferation index}

$\mathrm{Ki}-67$ showed as brown-yellow particles in the nuclei after 


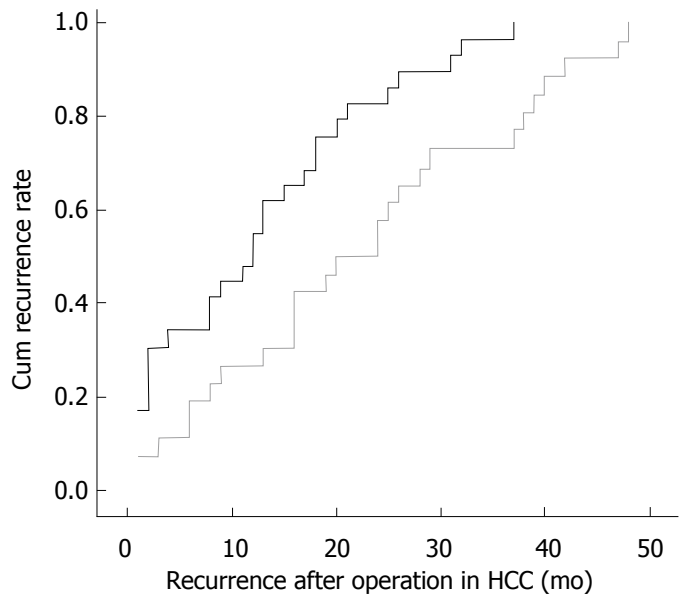

Figure 2 Correlation between survivin expression and recurrence rate of HCC after hepatectomy.

staining. Ki-67 labeling index in survivin-positive cancer was $33.83 \% \pm 18.90 \%$, while it was $19.60 \% \pm 19.35 \%$ in negative tumor. The difference was significantly different $(P<0.05)$. This suggests that the expression of survivin may promote the proliferation of HCC.

\section{Relationship between expression of survivin and recurrence and prognosis of $\mathrm{HCC}$}

The 1- and 3-year recurrence rates in survivin-positive HCC were $55.17 \%$ and $96.55 \%$, respectively, while the rates were $26.91 \%$ and $73.08 \%$, respectively, in survivin-negative HCC after hepatectomy. The recurrent time of survivin-positive HCC was significantly advanced $(P<0.05$, Figure 2$)$. Furthermore, the 1 - and 3-year survival rates in survivinpositive HCC were $58.62 \%$ and $10.34 \%$ after hepatectomy, respectively, but for survivin-negative HCC, the rates were $76.92 \%$ and $30.71 \%$, respectively. The 1 - and 3 -year survival rates were significantly lower in patients with survivin-positive HCC than those in survivin-negative HCC $(P<0.05$, Figure 3$)$. The expression of survivin may be used as an indicator for prognosis of HCC.

\section{DISCUSSION}

Among the recently described IAP family, survivin is characterized by a unique structure with a single BIR and no zinc-binding domain ${ }^{[1]}$, and is undetectable in terminally differentiated adult tissues, but becomes notably expressed in the most common human cancers, including esophageal, stomach, colorectal, breast and pancreatic carcinoma ${ }^{[12-16]}$. Survivin has also been implicated in the control of cell-cycle kinetics and inhibition of $\operatorname{apoptosis}^{[17-19]}$.

In our current study, we demonstrated that the expression of survivin was detected in $52.7 \%$ of patients with HCC, mainly localized in the cytoplasm of the carcinoma cells, with rare appearance in the nucleus. On the other hand, there have also been reports of a nuclear presence of survivin in $\mathrm{HCC}^{[20,21]}$. In previous studies, immunohistochemical analysis or RT-PCR of surgically resected tissues has revealed that approximately 30\%-90\% of HCC are positive for survivin expression ${ }^{[22-26]}$. The

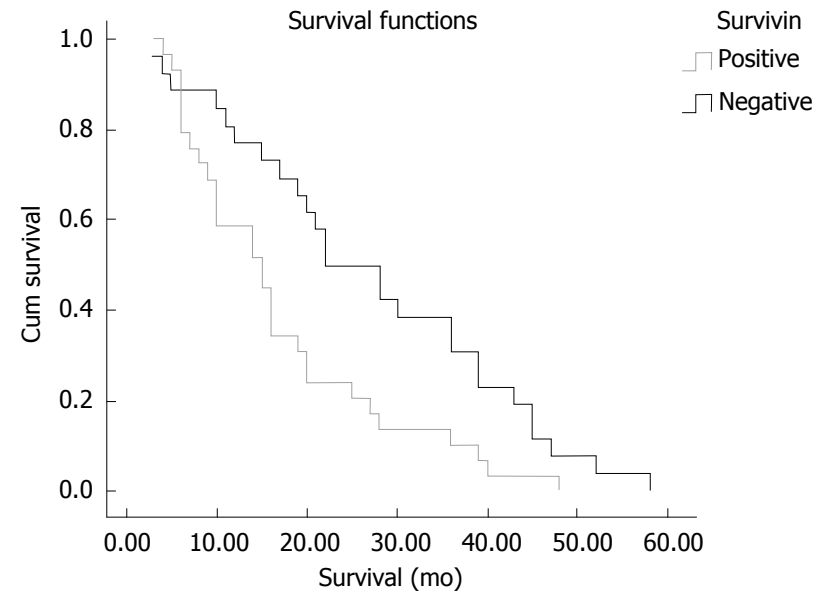

Figure 3 Kaplan-Meier curves for overall survival rates of patients with HCC according to survivin expression.

reasons for the difference may be the following: during the cell-division cycle, mRNA expression for survivin is extremely low in the G1 phase, and in the S phase is six times higher, while in the G2/M phase, the expression level of survivin increases suddenly to be 40 times higher than that in $G 1^{[27]}$. Therefore, the tumor cells in the $\mathrm{G} 1 / \mathrm{S}$ phase may represent negative expression, which would lead to different expression rate of survivin in different study. On the other hand, different criteria for positive expression of survivin or experimental methods may explain the different expression rates of survivin.

$\mathrm{Ki}-67$ is considered to be more useful for the determination of the proliferative activity of $\mathrm{HCC}$, and is known as a risk factor for HCC. In our study, we surprisingly found that expression of the proliferation index in survivin-positive HCC was higher than that in survivin-negative HCC. The results suggest that survivin may promote cell proliferation, and contribute to the development of HCC. Survivin may interact with the cell-cycle regulator $\mathrm{Cdk} 4$, which leads to $\mathrm{Cdk} 2 /$ cyclin $\mathrm{E}$ activation and $\mathrm{Rb}$ phosphorylation. As a result of survivin/ Cdk4 complex formation, p21 is released from its complex with Cdk4 and interacts with mitochondrial procaspase-3 to suppress Fas-mediated cell death. Survivin can also inhibit the activity of caspase-3/7 directly or indirectly, and this results in the generation and development of $\mathrm{HCC}^{[28-30]}$. The up-regulated expression of the proliferation index in survivin-positive HCC also suggests that survivin plays an important role in tumor progression.

The survivin expression in HCC was significantly correlated with portal vein thrombi and intrahepatic metastatic nodes. Therefore, survivin may play an important role in the development of HCC. Compared to survivin-negative HCC, survivin-positive HCC had a higher recurrent rate and lower 1- and 3-year survival rates. Survivin expression may play a role in the tumor biological characteristics of HCC, and may be a prognostic factor after hepatectomy. The study by Ikeguchi et al has also shown that high expression of survivin is associated with high recurrence and low survival rates ${ }^{[23]}$. Normal shedding of cells initiates the apoptosis process, but the over-expression of survivin exerts an anti-apoptotic effect, 
which leads to a high rate of cell proliferation. Therefore, survivin may play an important role in the progression of HCC and may facilitate metastatic spread via the blood stream.

In conclusion, survivin expression in HCC was significantly correlated with portal vein thrombi and intrahepatic metastatic nodes. There was a significant positive correlation between survivin expression and proliferation index. Survivin plays an important role in HCC progression through promoting cell proliferation, and may be a prognostic marker for HCC.

\section{COMMENTS}

\section{Background}

Survivin is a recently described member of the family of inhibitor of apoptosis proteins (IAPs). It has been shown that survivin is strongly associated with apoptosis, cell proliferation and cell-cycle control, and becomes markedly expressed in the most common human cancers.

\section{Research frontiers}

Immunohistochemical staining of survivin and Ki-67 was performed by the standard streptavidin-peroxidase (SP) technique for paraffin sections of hepatocellular carcinoma ( $\mathrm{HCC})$ tissues.

\section{Innovations and breakthroughs}

We demonstrated that the positive rate of survivin in $\mathrm{HCC}$ was $52.7 \%$, and a significant correlation was found between survivin expression and portal vein thrombi and intrahepatic metastatic nodes. The recurrence rate in survivin-positive $\mathrm{HCC}$ was significantly higher than that in survivin-negative $\mathrm{HCC}$ after hepatectomy. The 1- and 3-year survival rates of patients with survivin- positive tumors were significantly lower than those in patients with survivin-negative tumors. The proliferation index (Ki-67) in survivin-positive HCC was significantly higher than that in survivin-negative HCC.

\section{Applications}

Survivin may play an important role in progression of HCC by promoting cell proliferation, and may be positively correlated with a high risk of disease recurrence and poor prognosis in $\mathrm{HCC}$. Its expression can serve as a prognostic factor for patients with HCC after hepatectomy.

\section{Peer review}

This is an interesting correlative study of survivin expression and survival in a cohort of 55 patients. A significantly worse survival was observed in survivingpositive tumors. The data are of high quality.

\section{REFERENCES}

1 Thomas MB, Zhu AX. Hepatocellular carcinoma: the need for progress. J Clin Oncol 2005; 23: 2892-2899

2 Altieri DC, Marchisio PC. Survivin apoptosis: an interloper between cell death and cell proliferation in cancer. Lab Invest 1999; 79: 1327-1333

3 Giodini A, Kallio MJ, Wall NR, Gorbsky GJ, Tognin S, Marchisio PC, Symons M, Altieri DC. Regulation of microtubule stability and mitotic progression by survivin. Cancer Res 2002; 62: 2462-2467

4 Wakana Y, Kasuya K, Katayanagi S, Tsuchida A, Aoki T, Koyanagi Y, Ishii H, Ebihara Y. Effect of survivin on cell proliferation and apoptosis in gastric cancer. Oncol Rep 2002; 9: 1213-1218

5 Lu M, Kwan T, Yu C, Chen F, Freedman B, Schafer JM, Lee EJ, Jameson JL, Jordan VC, Cryns VL. Peroxisome proliferator-activated receptor gamma agonists promote TRAIL-induced apoptosis by reducing survivin levels via cyclin D3 repression and cell cycle arrest. J Biol Chem 2005; 280: 6742-6751

6 Kawasaki H, Toyoda M, Shinohara H, Okuda J, Watanabe
I, Yamamoto T, Tanaka K, Tenjo T, Tanigawa N. Expression of survivin correlates with apoptosis, proliferation, and angiogenesis during human colorectal tumorigenesis. Cancer 2001; 91: 2026-2032

7 Salz W, Eisenberg D, Plescia J, Garlick DS, Weiss RM, Wu $\mathrm{XR}$, Sun TT, Altieri DC. A survivin gene signature predicts aggressive tumor behavior. Cancer Res 2005; 65: 3531-3534

8 Sui L, Dong Y, Ohno M, Watanabe Y, Sugimoto K, Tokuda M. Survivin expression and its correlation with cell proliferation and prognosis in epithelial ovarian tumors. Int J Oncol 2002; 21: $315-320$

9 Caldas H, Jaynes FO, Boyer MW, Hammond S, Altura RA. Survivin and Granzyme B-induced apoptosis, a novel anticancer therapy. Mol Cancer Ther 2006; 5: 693-703

10 Ryan BM, Konecny GE, Kahlert S, Wang HJ, Untch M, Meng G, Pegram MD, Podratz KC, Crown J, Slamon DJ, Duffy MJ. Survivin expression in breast cancer predicts clinical outcome and is associated with HER2, VEGF, urokinase plasminogen activator and PAI-1. Ann Oncol 2006; 17: 597-604

11 Span PN, Tjan-Heijnen VC, Heuvel JJ, de Kok JB, Foekens JA, Sweep FC. Do the survivin (BIRC5) splice variants modulate or add to the prognostic value of total survivin in breast cancer? Clin Chem 2006; 52: 1693-1700

12 Ikeguchi M, Kaibara N. survivin messenger RNA expression is a good prognostic biomarker for oesophageal carcinoma. $\mathrm{Br}$ J Cancer 2002; 87: 883-887

13 Miyachi K, Sasaki K, Onodera S, Taguchi T, Nagamachi M, Kaneko H, Sunagawa M. Correlation between survivin mRNA expression and lymph node metastasis in gastric cancer. Gastric Cancer 2003; 6: 217-224

14 Sarela AI, Scott N, Ramsdale J, Markham AF, Guillou PJ. Immunohistochemical detection of the anti-apoptosis protein, survivin, predicts survival after curative resection of stage II colorectal carcinomas. Ann Surg Oncol 2001; 8: 305-310

15 Ryan B, O'Donovan N, Browne B, O'Shea C, Crown J, Hill AD, McDermott E, O'Higgins N, Duffy MJ. Expression of survivin and its splice variants survivin-2B and survivin-DeltaEx3 in breast cancer. Br I Cancer 2005; 92: 120-124

16 Sarela AI, Verbeke CS, Ramsdale J, Davies CL, Markham AF, Guillou PJ. Expression of survivin, a novel inhibitor of apoptosis and cell cycle regulatory protein, in pancreatic adenocarcinoma. Br J Cancer 2002; 86: 886-892

17 O'Connor DS, Grossman D, Plescia J, Li F, Zhang H, Villa A, Tognin S, Marchisio PC, Altieri DC. Regulation of apoptosis at cell division by p34cdc2 phosphorylation of survivin. Proc Natl Acad Sci USA 2000; 97: 13103-13107

18 Fukuda S, Mantel CR, Pelus LM. Survivin regulates hematopoietic progenitor cell proliferation through p21WAF1/Cip1-dependent and -independent pathways. Blood 2004; 103: 120-127

19 Rosa J, Canovas P, Islam A, Altieri DC, Doxsey SJ. Survivin modulates microtubule dynamics and nucleation throughout the cell cycle. Mol Biol Cell 2006; 17: 1483-1493

20 Morinaga S, Nakamura $Y$, Ishiwa N, Yoshikawa T, Noguchi Y, Yamamoto Y, Rino Y, Imada T, Takanashi Y, Akaike M, Sugimasa $Y$, Takemiya S. Expression of survivin mRNA associates with apoptosis, proliferation and histologically aggressive features in hepatocellular carcinoma. Oncol Rep 2004; 12: 1189-1194

21 Ito T, Shiraki K, Sugimoto K, Yamanaka T, Fujikawa K, Ito M, Takase K, Moriyama M, Kawano H, Hayashida M, Nakano $\mathrm{T}$, Suzuki A. Survivin promotes cell proliferation in human hepatocellular carcinoma. Hepatology 2000; 31: 1080-1085

22 Ikeguchi M, Ueta T, Yamane Y, Hirooka Y, Kaibara N. Inducible nitric oxide synthase and survivin messenger RNA expression in hepatocellular carcinoma. Clin Cancer Res 2002; 8: 3131-3136

23 Ikeguchi M, Ueda T, Sakatani T, Hirooka Y, Kaibara N. Expression of survivin messenger RNA correlates with poor prognosis in patients with hepatocellular carcinoma. Diagn Mol Pathol 2002; 11: 33-40

24 Bao ST, Gui SQ, Lin MS. Relationship between expression of Smac and Survivin and apoptosis of primary hepatocellular carcinoma. Hepatobiliary Pancreat Dis Int 2006; 5: 580-583 
25 Fields AC, Cotsonis G, Sexton D, Santoianni R, Cohen C. Survivin expression in hepatocellular carcinoma: correlation with proliferation, prognostic parameters, and outcome. Mod Pathol 2004; 17: 1378-1385

26 Ikeguchi M, Ueta T, Yamane Y, Hirooka Y, Kaibara N. Quantitative analysis of heparanase messenger RNA expression in hepatocellular carcinoma. J Surg Oncol 2002; 81: 148-154; disscusion 154

27 Beardmore VA, Ahonen LJ, Gorbsky GJ, Kallio MJ. Survivin dynamics increases at centromeres during G2/M phase transition and is regulated by microtubule-attachment and Aurora B kinase activity. J Cell Sci 2004; 117: 4033-4042
28 Shin S, Sung BJ, Cho YS, Kim HJ, Ha NC, Hwang JI, Chung $\mathrm{CW}$, Jung $\mathrm{YK}, \mathrm{Oh} \mathrm{BH}$. An anti-apoptotic protein human survivin is a direct inhibitor of caspase-3 and -7. Biochemistry 2001; 40: 1117-1123

29 Dai DJ, Lu CD, Lai RY, Guo JM, Meng H, Chen WS, Gu J. Survivin antisense compound inhibits proliferation and promotes apoptosis in liver cancer cells. World J Gastroenterol 2005; 11: 193-199

30 Rödel F, Hoffmann J, Distel L, Herrmann M, Noisternig T, Papadopoulos T, Sauer R, Rödel C. Survivin as a radioresistance factor, and prognostic and therapeutic target for radiotherapy in rectal cancer. Cancer Res 2005; 65: 4881-4887

S- Editor Zhu LH L- Editor Kerr C E- Editor Yin DH 